\title{
VACUUM DECAYING COSMOLOGICAL MODELS AND GRAVITATIONAL LENSING
}

\author{
I. WAGA ${ }^{1,2}$ AND L. F. BLOOMFIELD TORRES ${ }^{2}$ \\ 1 NASA/Fermilab Astrophysics Center \\ Fermi National Accelerator Laboratory, Batavia, IL 60510 \\ 2 Universidade Federal do Rio de Janeiro, \\ Instituto de Física \\ Rio de Janeiro - RJ - Brasil -21943
}

We investigate the statistical properties of gravitational lenses for flat models in which a cosmological term decreases with time as $\Lambda \propto a^{-m}$, where $a$ is the scale factor and $m$ is a parameter $(0 \leq m<3)$ (Bloomfield Torres and Waga 1995).

We use two different approximations in our computations. In the first one, that we call "A1", we follow Fukugita and Turner (1991) and use a simplified approach to take into account magnification bias (as in Fukugita \& Peebles 1995). In the second approach, named "A2", we model E/SO galaxies as singular isothermal spheres, use a velocity dispersion of $\sigma_{\star}=220$ $\mathrm{km} \mathrm{s}^{-1}$ (no correction factor), and the magnification bias is computed with the Wallington and Narayan (1993) quasar luminosity function (QLF).

In Table 1 we display, for three flat models and for the open FRW model with $\Omega_{m 0}=0.2$ (model D), the predicted number of lensed quasars for the Hubble Space Telescope (HST) Snapshot Survey (Maoz et al. 1993). By assuming Poisson statistics we also display, for all cases, the probability of detecting the observed number (four) of lensed quasars.

We applied Kochanek's (1993) maximum likelihood method to the flat models. Contours of constant likelihood $(95.4 \%, 68 \%$ and $50 \%$ confidence levels) are plotted in Figure 1. If $m \simeq 0$, regions with a high value of $\Lambda$ have low likelihood. However if, for instance, $m \gtrsim 1$ and $\Omega_{m 0} \gtrsim 0.2$ or $m \gtrsim 1.5$ this constraint does not exist and models with low $\Omega_{m 0}$ have high likelihoods for reproducing the observed lens statistics in the HST Snapshot Survey. 
TABLE 1. Predicted number of lensed quasars for the HSTSS and model probabilities

\begin{tabular}{ccccc} 
& $\begin{array}{c}\text { model A } \\
k=0 \\
\Omega_{m 0}=1\end{array}$ & $\begin{array}{c}\text { model B } \\
k=0, m=2 \\
\Omega_{m 0}=0.2\end{array}$ & $\begin{array}{c}\text { model C } \\
k=0, m=0 \\
\Omega_{m 0}=0.2\end{array}$ & $\begin{array}{c}\text { model D } \\
\Omega_{m 0}=-1\end{array}$ \\
\hline "A1" & 2.6 & 4.7 & 10.7 & 4.1 \\
probability & $18 \%$ & $17 \%$ & $0.3 \%$ & $19 \%$ \\
"A2" & 3.2 & 5.7 & 12.7 & 5.1 \\
probability & $20 \%$ & $12 \%$ & $0.03 \%$ & $15 \%$ \\
\hline
\end{tabular}
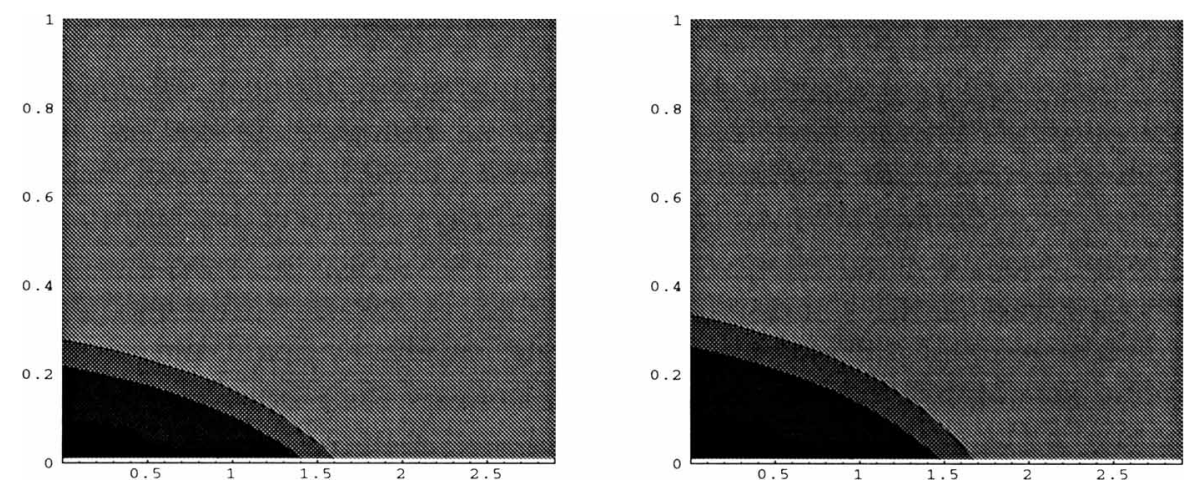

Figure 1. Contours of constant likelihood (95.4\%,68\% and 50\% confidence levels) for flat models are plotted in the $\Omega_{m 0}$ (y-axis) and $m$ (x-axis) parameter space. Regions with larger likelihood are represented by lighter shades. The left panel was obtained following Fukugita and Turner (1991) and Fukugita and Peebles (1995). For the right panel we used the Wallington and Narayan (1993) QLF.

Acknowledgements: We would like to thank Chris Kochanek for several discussions on lens statistics, core radius, magnification bias and other selection effects. This work was supported in part by the Brazilian agency CNPq and by the DOE and NASA at Fermilab through grant NAG5-2788.

\section{References}

Bloomfield Torres, \& L. F., Waga, I., 1995, submitted to MNRAS

Fukugita, M., \& Peebles, P. J. E., 1995, submitted to ApJL

Fukugita, M., \& Turner, E.L., 1991, MNRAS, 253, 99

Kochanek, C. S., 1993, ApJ, 419, 12

Maoz, D., et al., 1993, ApJ, 409, 28

Wallington, S., \& Narayan, R., 1993, ApJ, 403, 517 